MS101.P12

\title{
Silver porphyrin molecular film alcohol gas sensor
}

Supakorn Boonyuen ${ }^{1}$, Monta Malaithong ${ }^{1}$, Tossapon Prohmsatit ${ }^{1}$, Sumana Kladsomboon ${ }^{2}$

${ }^{1}$ Department Of Chemistry, Faculty Of Science And Technology, Thammasat University, Patumthani, Thailand, ${ }^{2}$ Center for Research and Innovation, Faculty of Medical Technology, Mahidol University, Nakhon Pathom 73170, Thailand, Nakhon Pathom, Thailand

E-mail: chemistrytu@gmail.com

A new 5,10,15,20-tetrakis(4-butyloxy)phenyl porphyrinatosilver(II) [Ag-TOBPP] compound has been successfully synthesized and characterized. The structure of the synthesized compounds was confirmed by Infrared Spectroscopy (IR), Mass Spectroscopy (MS), Fluorescence and UV-Vis absorption spectra. In this work, [Ag-TOBPP] was modified for detecting specific alcoholic volatile organic compounds (VOCs) by dissolving the complex in dichloromethane and coated onto clean glass slides at ambient conditions. Methanol ( $\mathrm{MeOH})$, ethanol (EtOH) and isopropanol (PrOH) vapors were selected as samples which were detected by Electronic nose (E-nose). Principal component analysis (PCA) was used as a pattern recognition method to analyze the data set produced from an UV-Vis spectrophotometer. The result shows that [Ag-TOBPP] organic thin film can perform efficiently as a selective alcohol sensor.

1. Lyutakov, O.H.; Solovyez, A.; Kalachyova, Y.; Svorcik, V. RSC Adv 2014, 4, 50624-50630.

2. Fasalu, K.; Subramaniam, S.; Chellaiah, A. Polyhedron 2015, 97, 66-74.

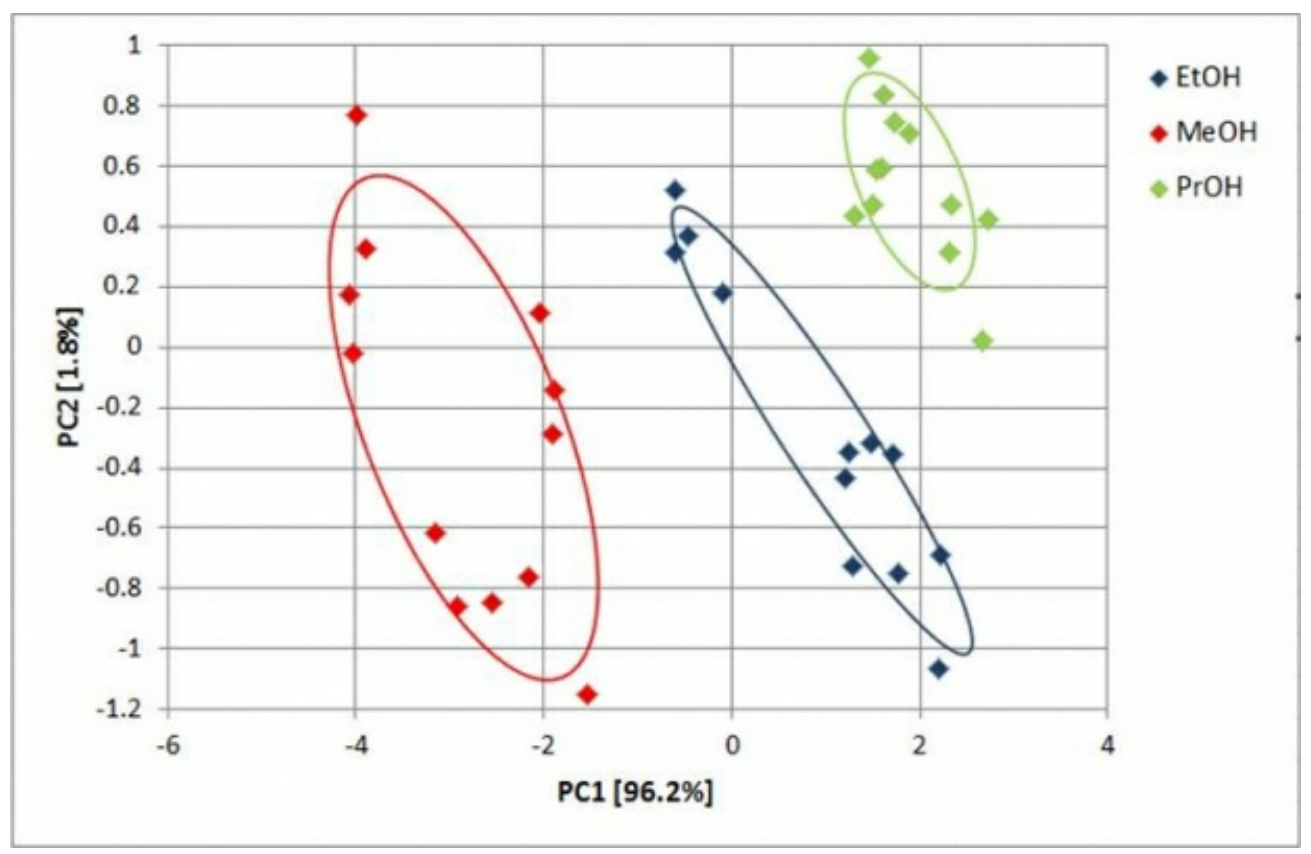

Keywords: Metallo-porphyrin; Alcohol detection; Electronic nose 